\title{
Functional characterization of TRICHOMELESS2, a new single-repeat R3 MYB transcription factor in the regulation of trichome patterning in Arabidopsis
}

Lijun Gan ${ }^{1,4}$, Kai Xia ${ }^{1}$, Jin-Gui Chen ${ }^{3,4}$ and Shucai Wang ${ }^{2,4^{*}}$

\begin{abstract}
Background: Single-repeat R3 MYB transcription factors (single-repeat MYBs) play important roles in controlling trichome patterning in Arabidopsis. It was proposed that single-repeat MYBs negatively regulate trichome formation by competing with GLABRA1 (GL1) for binding GLABRA3/ENHANCER OF GLABRA3 (GL3/EGL3), thus inhibiting the formation of activator complex TTG1(TRANSPARENT TESTA GLABRA1)-GL3/EGL3-GL1 that is required for the activation of GLABRA2 (GL2), whose product is a positive regulator of trichome formation. Previously we identified a novel single-repeat MYB transcription factor, TRICHOMELESS1 (TCL1), which negatively regulates trichome formation on the inflorescence stems and pedicels by directly suppressing the expression of GL1.

Results: We analyzed here the role of TRICHOMELESS2 (TCL2), a previously-uncharacterized single-repeat MYB transcription factor in trichome patterning in Arabidopsis. We showed that TCL2 is closely related to TCL1, and like TCL1 and other single-repeat MYBs, TCL2 interacts with GL3. Overexpression of TCL2 conferred glabrous phenotype while knockdown of TCL2 via RNAi induced ectopic trichome formation on the inflorescence stems and pedicels, a phenotype that was previously observed in tcl1 mutants. These results suggested that TCL2 may have overlapping function with TCL1 in controlling trichome formation on inflorescences. On the other hand, although the transcription of TCL2, like TCL1, is not controlled by the activator complex formed by GL1 and GL3, and TCL2 and TCL1 proteins are more than $80 \%$ identical at the amino acid level, the expression of TCL2 under the control of TCL1 promoter only partially recovered the mutant phenotype of tcl1, implying that TCL2 and TCL1 are not fully functional equivalent.

Conclusions: TCL2 function redundantly with TCL1 in controlling trichome formation on inflorescences, but they are not fully functional equivalent. Transcription of TCL2 is not controlled by activator complex formed by GL1 and GL3, but MIR156 controlled SQUAMOSA PROMOTER BINDING PROTEIN LIKE (SPL) transcription factors. However, SPLs might require co-activators to regulate the expression of their target genes, including TCL1, TRY and possibly, TCL2.
\end{abstract}

\section{Background}

Trichome patterning in Arabidopsis is controlled by several different types of transcription factors. Based on available evidence, it was proposed that an R2R3 MYBtype transcription factor GLABRA1 (GL1) [1], a bHLH transcription factors GLABRA3 (GL3) [2] or ENHANCER OF GLABRA3 (EGL3) [3], and a WD40-repeat protein TRANSPARENT TESTA GLABRA1 (TTG1) $[4,5]$, form an activator complex TTG1-GL3/EGL3-GL1. This

\footnotetext{
* Correspondence: wangshucai@yahoo.com

${ }^{2}$ Key Laboratory of Molecular Epigenetics of MOE and Institute of Genetics \& Cytology, Northeast Normal University, Changchun 130024, China Full list of author information is available at the end of the article
}

activator complex activates the expression of GLABRA2 (GL2), which encodes a homeodomain protein that promotes trichome formation in shoots [6-8]. The same activator complex also induces the expression of some single-repeat R3 MYB genes.

So far a total of six genes in the Arabidopsis genome have been reported encoding single-repeat R3 MYB transcription factors, including TRIPTYCHON (TRY) [9,10], CAPRICE (CPC) [11,12], TRICHOMELESS1(TCL1) [13], and ENHANCER OF TRY AND CPC1, 2 and 3 (ETC1, ETC2 and ETC3/CPL3) [14-18]. Single-repeat MYB transcription factors are characterized by their short sequence (75-112 amino acids) and consist largely of the 
single MYB domain [19]. Single-repeat R3 MYBs can move from a trichome precursor cell to its neighboring cells to block the formation of the activator complex by competing with GL1 in binding GL3 or EGL3, thus inhibiting trichome formation in shoots $[8,13,18,19]$. These single-repeat R3 MYB transcription factors differ in their binding strength to GL3 and their capacity to compete with GL1 for binding GL3. A yeast three-hybrid assays suggested that CPC is the most potent inhibitor followed by ETC1, TRY, ETC3 and ETC2 [18]. A protoplast transfection assay showed that TCL1 is stronger than CPC in their ability to bind GL3 [19].

Although over expressing any of these six single-repeat R3 transcription factors in Arabidopsis causes a glabrous phenotype, single mutants of these genes have different phenotypes. Mutation in TRY results in trichome cluster phenotype $[9,10]$, mutation in $C P C$ increases trichome number [11,12], and mutation in TCL1 causes ectopic trichome formation on inflorescence stems and pedicels [13]. Mutation in ETC1, ETC2 or ETC3 does not dramatically affect trichome formation. However, analysis of double, triple and higher order mutants between these single mutants indicated that all six single-repeat MYB transcription factors function in a highly redundant manner to control trichome formation in Arabidopsis [13-18,20].

In addition to competing with GL1 for binding GL3, our previous results showed that TCL1 could also directly suppress the expression of GL1 [13]. Interestingly, unlike many other single-repeat MYBs, the expression of TCL1 is not controlled by the activator complex formed by GL1 and GL3/EGL3. Recently, it has been found that microRNA156 (MIR156)-targeted SQUAMOSA PROMOTER BINDING PROTEIN LIKE (SPL) 9 can activate TCL1 and TRY expression through binding to their promoters [21].

Here we analyzed the role of TRICHOMELESS2 (TCL2), a previously uncharacterized single-repeat MYB transcription factor in trichome patterning in Arabidopsis. We demonstrated that TCL2, like other single-repeat MYBs, negatively regulates trichome formation. Overexpression of TCL2 conferred glabrous phenotype while knockdown of TCL2 via RNAi induced ectopic trichome formation on inflorescence stems and pedicels, a phenotype that was previously observed in tcl1 mutants. Furthermore we provide genetic evidence that TCL2 and TCL1 may not be fully functional equivalent. We also showed that MIR156 is involved in the regulation of TCL2, and SPLs may require co-activators to regulate the expression of their target genes, including TCL1, TRY, and possibly, TCL2.

\section{Results}

\section{TRICHOMELESS2 is closely related to TCL1}

In Arabidopsis, a total of six single-repeat MYB transcription factors have been characterized so far [13]. In order to examine whether there is any uncharacterized singlerepeat MYB transcription factors, the entire amino acid sequence of TCL1 was used as a template to BLAST Arabidopsis protein database (http://www.ncbi.nlm.nih.gov). In addition to TRY, CPC, TCL1, ETC1, ETC2 and ETC3/ $C P L 3$ that have been reported previously, our analysis revealed that this is another single-repeat MYB transcription factor encoded by At $2 g 30424$ in the Arabidopsis genome. The gene encoding this uncharacterized singlerepeat MYB was designated TRICHOMELESS2 (TCL2). TCL2 is nearby two other single-repeat MYB genes, ETC2 and TCL1, on chromosome II (Figure 1A), with $E T C 2$ is a tandem repeat gene with TCL2, while TCL2 and TCL1 is separated by another gene At2g30430. Phylogenic analysis using full length protein sequences showed that TCL2 is closely related to TCL1 (Figure 1B). TCL2 shares $80 \%$ identify with TCL1 at the amino acid level. As shown in Figure 1C, like TCL1 and other singlerepeat MYBs, TCL2 also contains the conserved amino acid signature $[\mathrm{D} / \mathrm{E}] \mathrm{L} \times 2[\mathrm{R} / \mathrm{K}] \times 3 \mathrm{~L} \times 6 \mathrm{~L} \times 3 \mathrm{R}$ that is required for the interaction with $\mathrm{R} / \mathrm{B}$-like bHLH transcription factors[22] and the conserved amino acids that have been shown to be required for the cell-to-cell movement of the single-repeat MYB transcription factor CPC [23].

\section{Expression and subcellular localization of TCL2}

To characterize the function of TCL2, we first examined the expression of TCL2 across various tissues and organs. We also compared the expression pattern of TCL2 with that of TCL1 because TCL2 is more closely related to TCL1 than other single-repeat MYBs at the amino acid level. Various tissues and organs of wild-type plants were harvested, and RT-PCR was used to examine the expression of TCL2 and TCL1. We found that TCL2 is expressed in all tissues/organs tested except young siliques, where TCL1 is highly expressed (Figure 2). TCL2 is also highly expressed in cotyledons, organs that normally do not produce any trichomes and where the transcript of TCL1 was at undetectable level (Figure 2). To get more details about when, where and in response to what stimulus TCL2 is expressed, we then searched public available gene expression database genevestigator (http://www.genevestigator. com/gv) using At2g30424 as gene ID, however, results showed that there is no probeset could be found for the gene ID provided, possibly because TCL2 is previously unidentified gene, and no probes for this gene have been printed on microarrays used for all those experiments that provided data for the database.

To examine TCL2's subcellular localization, we generated transgenic plants expressing TCL2-GFP fusion protein under the control of TCL2's own promoter, a genomic DNA fragment that covers the region -1502 to +1 of the start codon of TCL2. The transgenic plant 


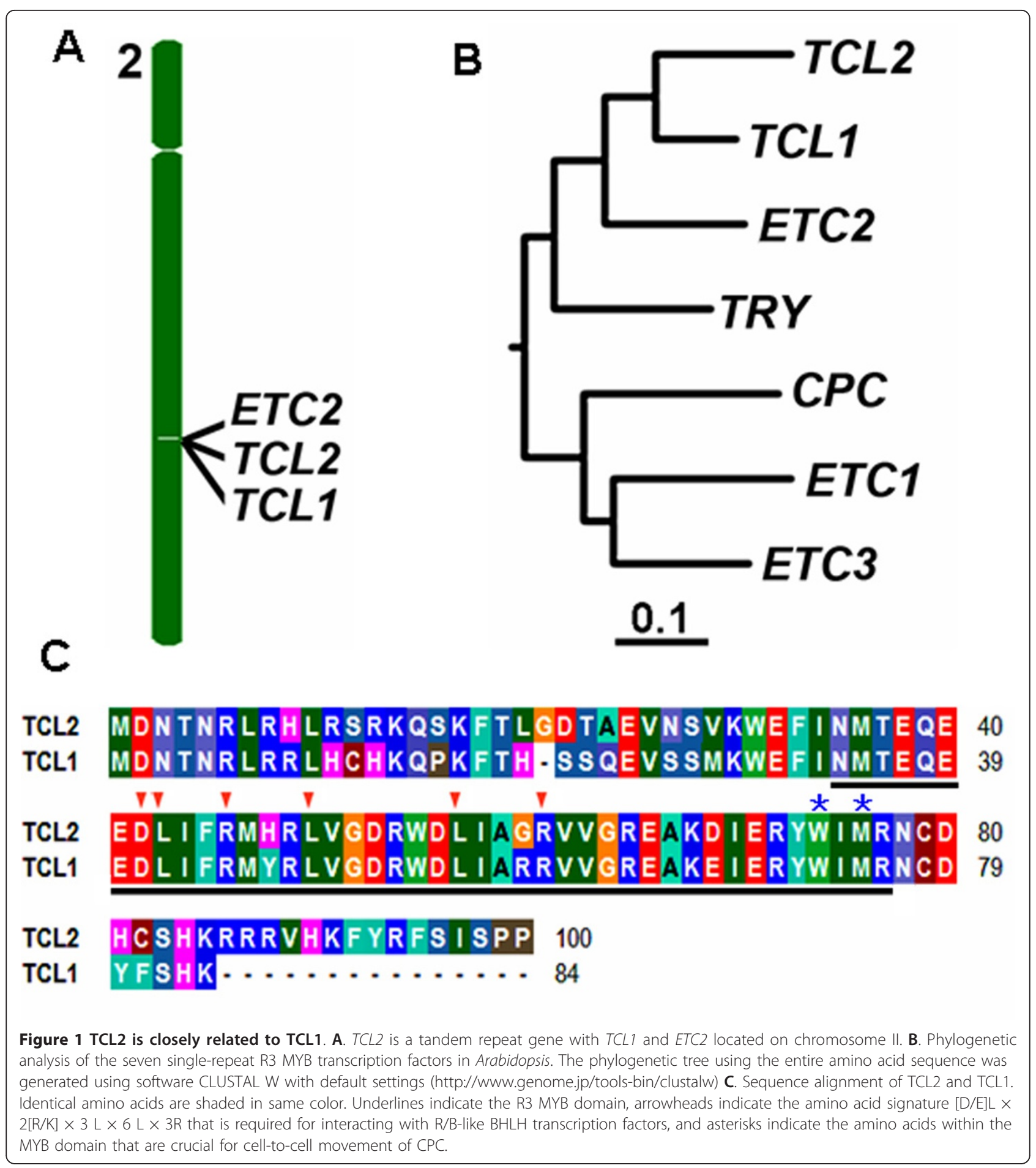

produces reduced number of trichomes (Figure 3A), a phenotype similar to that of PTCL1:TCL1-GFP transgenic plant [13],indicating that the reduction in trichome number is due to an increase on the copy number of the TCL2 gene, as overexpression of TCL2 showed a greatly reduced trichome phenotype (Figure 4). These results also indicate that both the TCL2 promoter and TCL2-GFP fusion protein are likely functional. By using the transgenic plants obtained, we showed that TCL2-GFP proteins are mainly observed in the nucleus of epidermal cells (Figure 3B).

TCL2 is a negative regulator of trichome formation To analyze the function of TCL2 in trichome formation, we generated transgenic plants overexpressing HA 


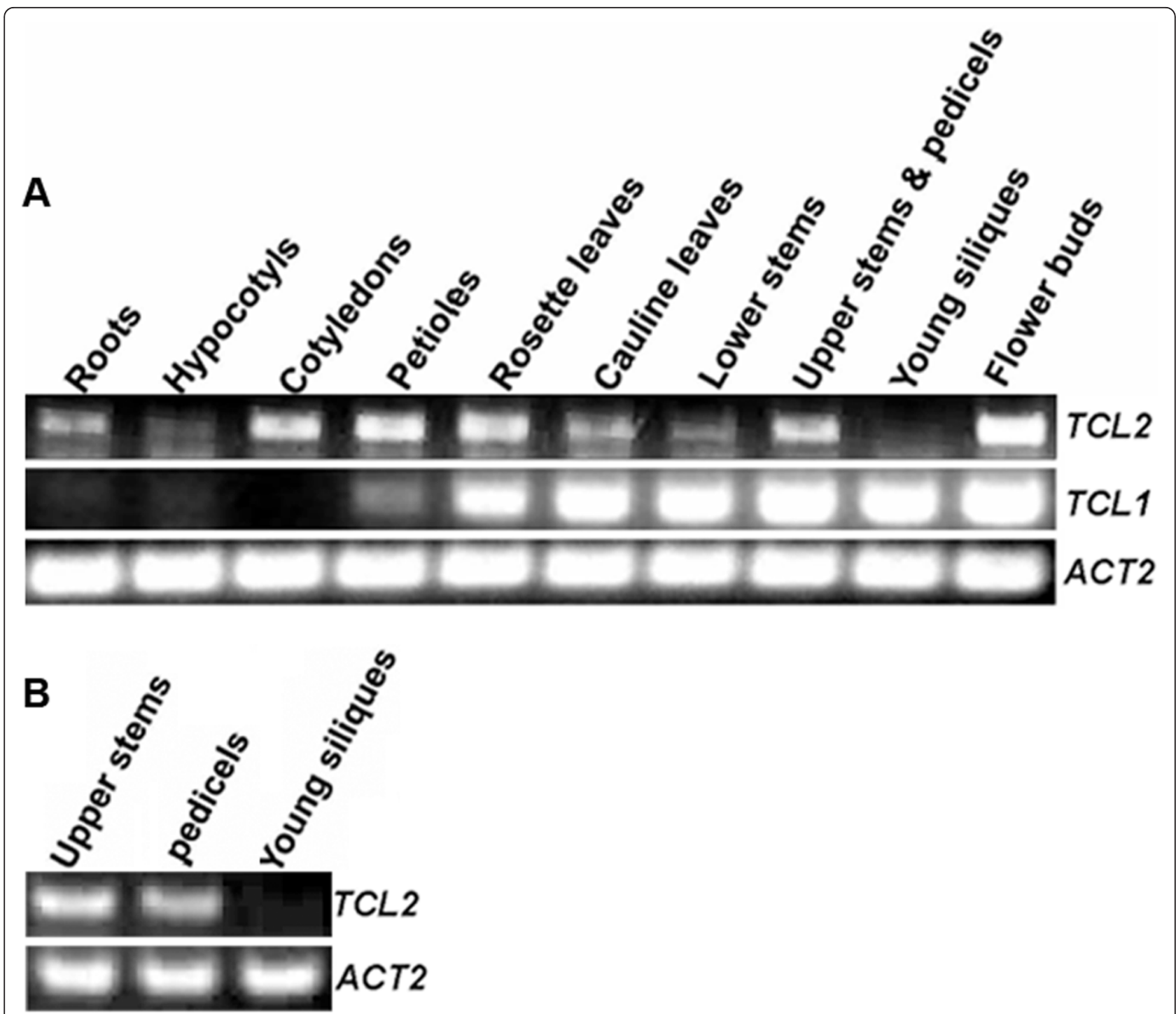

Figure 2 Expression of TCL2 and TCL1 in Arabidopsis. A. Expression of TCL2 and TCL1 in different tissues of Arabidopsis. B. Expression of TCL2 in pedicels. Tissues from different parts of wild-type Arabidopsis were collected, and RNA was isolated, then RT-PCR was used to check the expression of TCL2 and TCL1. The expression of ACTIN2 (ACT2) was used as a control.

tagged TCL2 under the control of $35 S$ promoter (35S: $H A-T C L 2)$. Transcript levels of TCL2 were examined by RT-PCR using HA-specific and TCL2 specific primers (Figure 4A). As observed previously for all other singlerepeat R3 MYB transcription factors, transgenic plants overexpressing TCL2 resulted in glabrous phenotypes, with no trichome formation on rosette leaves, inflorescence stems, cauline leaves or floral organs (Figure 4B).

To further analyze the function of TCL2 in trichome formation, we sought loss-of-function alleles of TCL2. By searching the SALK T-DNA Express Database, we found there are four T-DNA insertion mutants that are related to TCL2. However, in all those four lines, the T-DNA was inserted in the promoter regions, and expression of TCL2 is largely unaffected as examined by RT-PCR (data not shown). As a result, all the mutants have wild-type trichome phenotypes.

Therefore, we took RNAi approach to knock down the expression of TCL2. Since TCL2's ORF fragment is relative small, only 303 bp in length, we used the full-length ORF to create the RNAi construct. We selected three independent transgenic lines displaying ectopic trichome formation on inflorescence stems and pedicels (Figure 4B), similar to that of tcl1 mutant [13]. RT-PCR results showed that expression level of TCL2 in the transgenic lines was dramatically reduced (Figure 4A), while expression of 


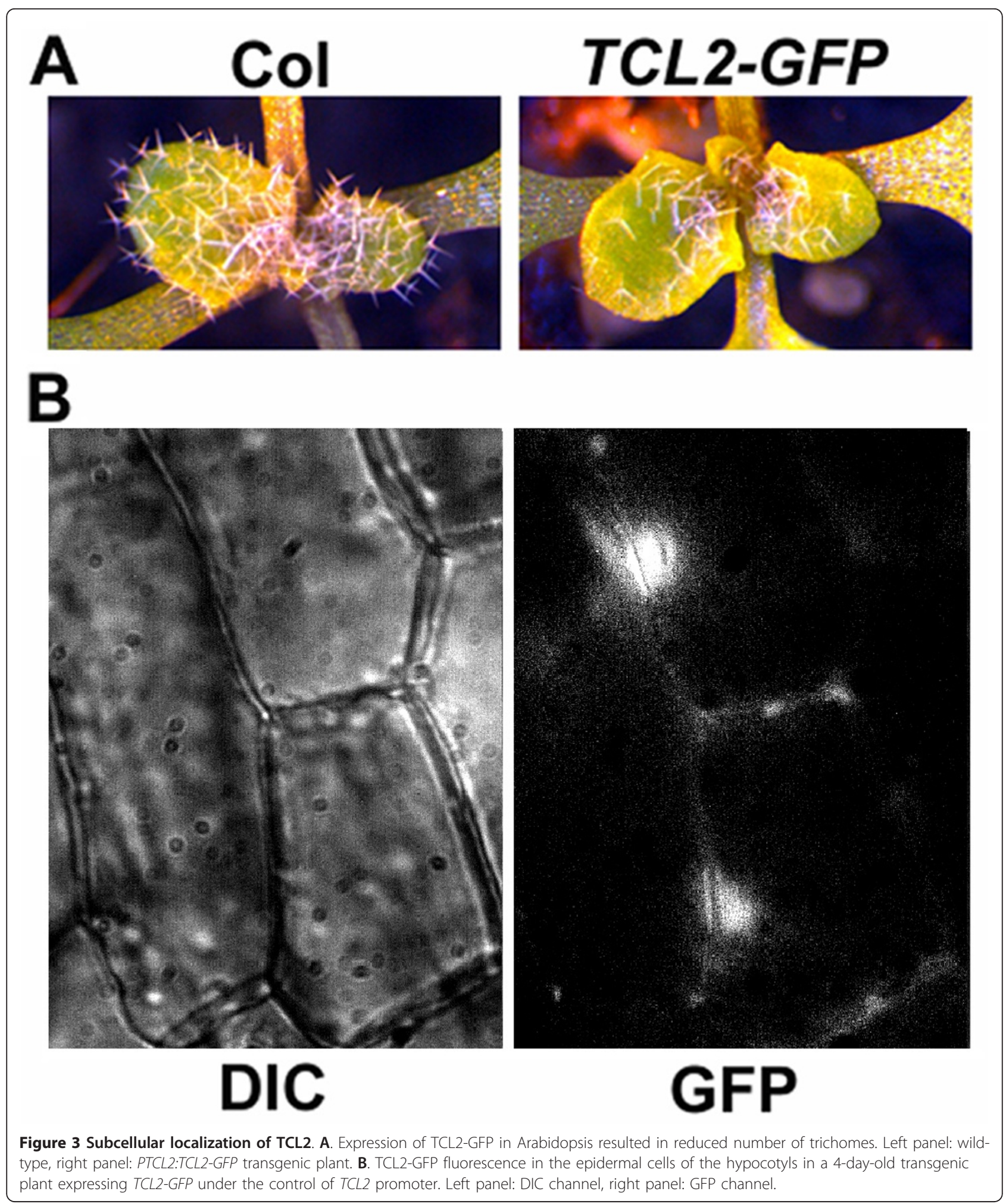

other single-repeat MYB genes was largely unaffected (see Additional file 1), indicating that the phenotype observed is caused by down-expression of TCL2.
When we quantified the number of trichomes on internodes and pedicels, we found that compared with tcll mutant, there are fewer internodes and pedicels 


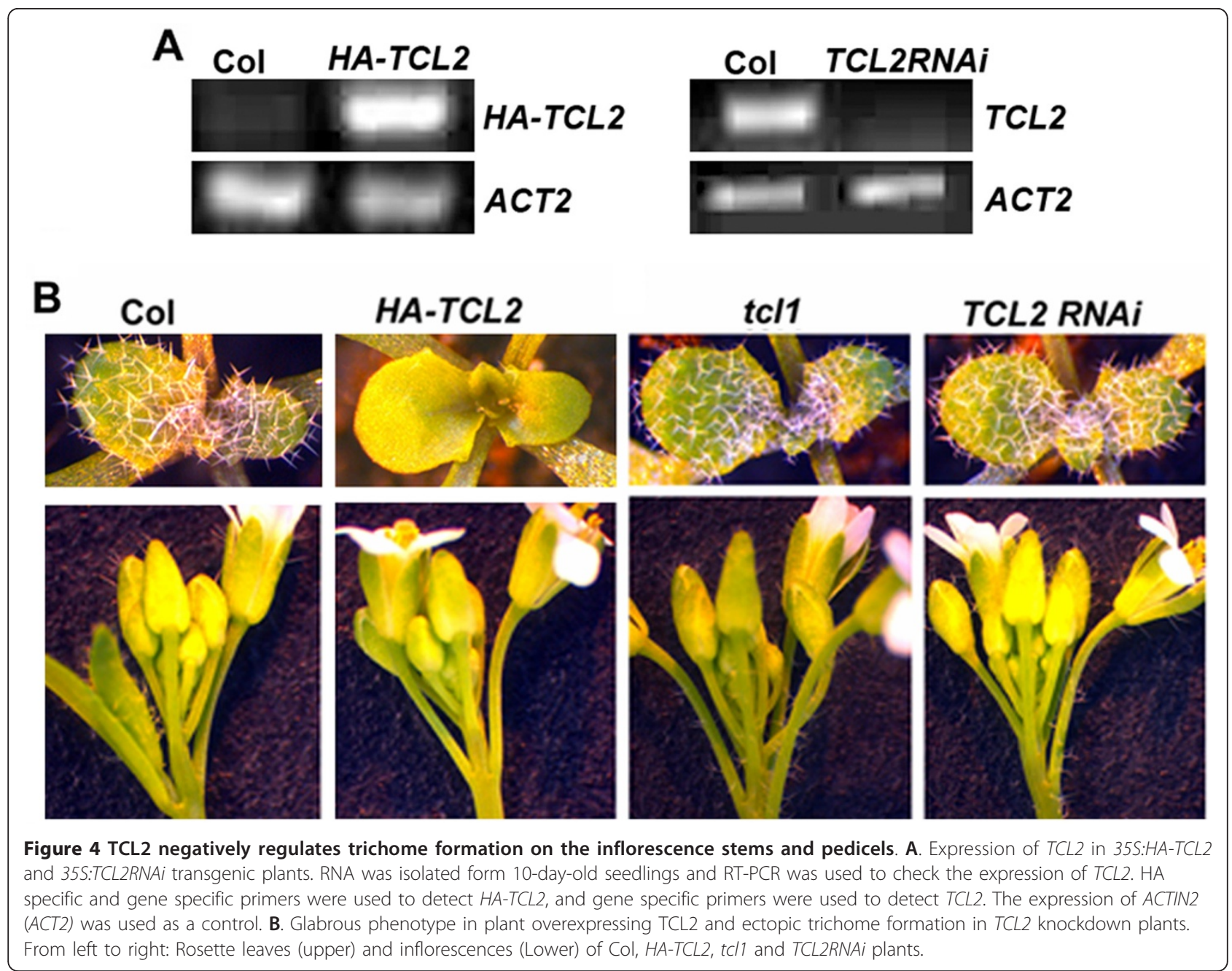

bearing ectopic trichomes in TCL2RNAi mutant (Figure $5)$.

TCL2 partially rescued tcl1 mutant trichome phenotypes Previously, we identified TCL1 as a major single-repeat MYB transcription factor that negatively regulates trichome formation on the inflorescences and pedicels [13]. The tcl1 mutant confers ectopic trichome formation on the inflorescence stems and pedicels. Here we showed that transgenic plants with reduced expression of TCL2 have similar phenotype as that of $t c l 1$ (Figure $4 \mathrm{~B}$, Figure 5). Loss-of-function mutations in any other single-repeat MYBs including TRY, CPC, ETC1, ETC2, and ETC3 do not result in similar inflorescence and pedicel trichome phenotypes as shown in tcll mutant or TCL2RNA $i$ lines. Therefore, we wanted to further examine the relationship between TCL2 and TCL1 in trichome formation on the inflorescence stems and pedicels. Double mutant was generated between $t c l 1$ and TCL2RNAi mutants, however, the double mutant was indistinguishable from tcl1 single mutant (data not shown), possibly because higher order redundancy function among single repeat genes [19]. Therefore we turned to test if TCL2 could rescue tcl1 mutant phenotype. Transgenic plants were generated to express TCL2 under the control of TCL1 promoter in a tcll background $\left(P_{T C L 1}: T C L 2 / t c l 1\right)$. Our previous results have showed that expression of TCL1-GFP fusion protein under the control of TCL1 promoter fully rescued tcl1 trichome phenotype [13], indicating that the TCL1 promoter used is fully functionally. As shown in Figure 5, expression of TCL2 protein under the TCL1 promoter only partially rescued $t c l 1$ mutant phenotype, indicating that TCL2 is functionally similar but not identical to TCL1 in controlling trichome patterning on the inflorescence stems and pedicels.

TCL2 interacts with GL3

It has been proposed that single-repeat MYB transcription factors control trichome formation by competing 


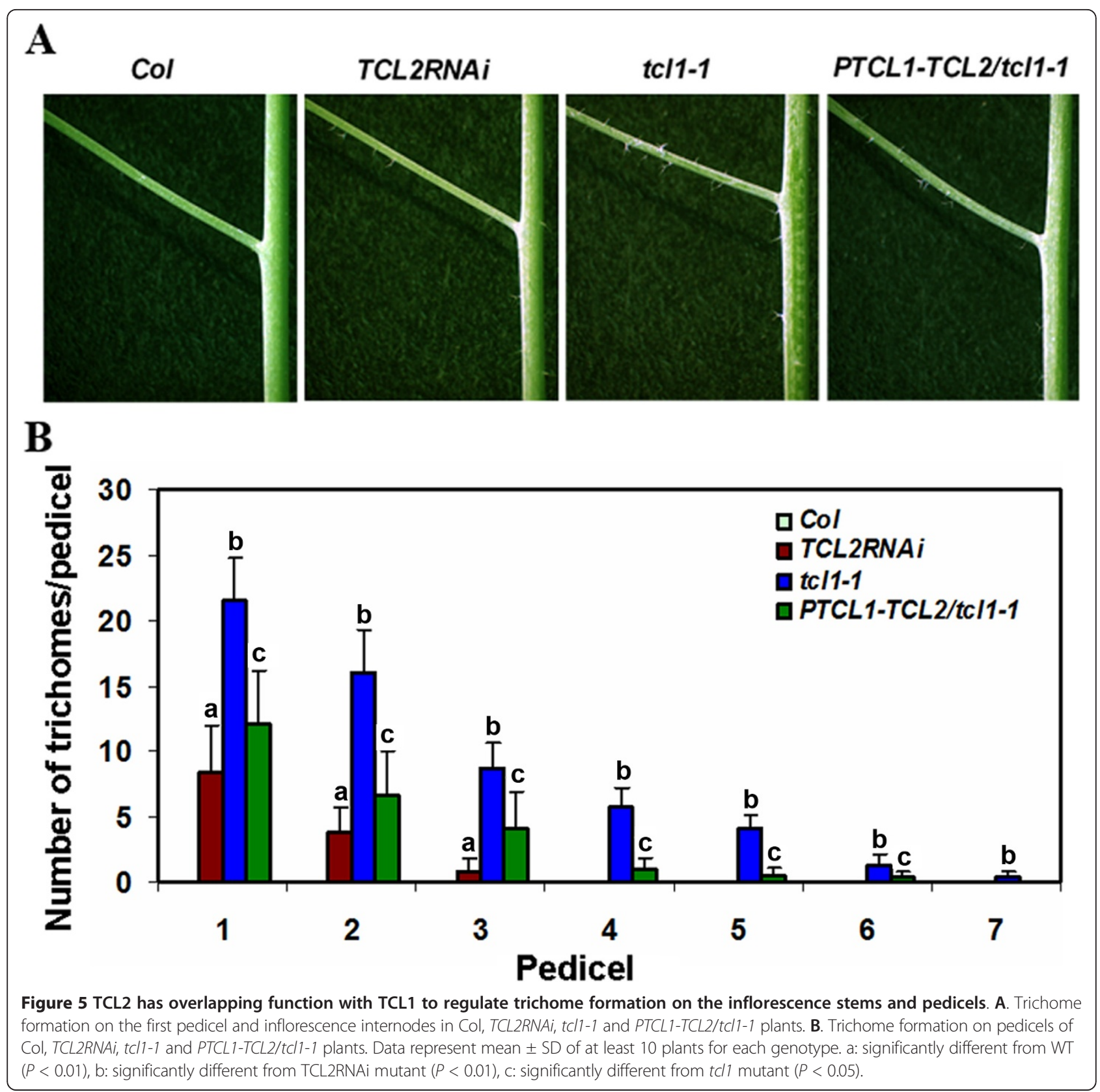

with GL1 for binding GL3, thus blocking the formation of TTG1-GL3-GL1 activator complex. We have previously demonstrated that TRY, CPC, TCL1, ETC1, ETC2, and ETC3, interact with GL3 using a protoplast transfection system [19]. To test if TCL2 controls trichome formation using a similar mechanism, we tested if TCL2 interacts with GL3 in plant cells.

A protoplast transient expression system was used to test the interaction between TCL2 and GL3. A Gal4GUS reporter, together with the effectors GL3 and a Gal4 DNA binding domain (GD) fused TCL2 (GDTCL2) were co-transfected into Arabidopsis protoplasts.
$G D$ alone and GD-TCL1 were used as negative and positive control, respectively. As shown in Figure 6A, with or without GL3, GD alone could not activate the expression of the reporter gene. In the absence of GL3, neither GD-TCL1 nor GD-TCL2 activated the expression of the reporter gene presumably due to the fact that singlerepeat R3 transcription factors do not have activation domains. However, in the presence of GL3, both GDTCL2 and GD-TCL1 activated the reporter gene, indicating that TCL2 interacts with GL3 in plant cells. It should be noted that GL3 can function as a transcriptional activator, however, alone it cannot be recruited to 


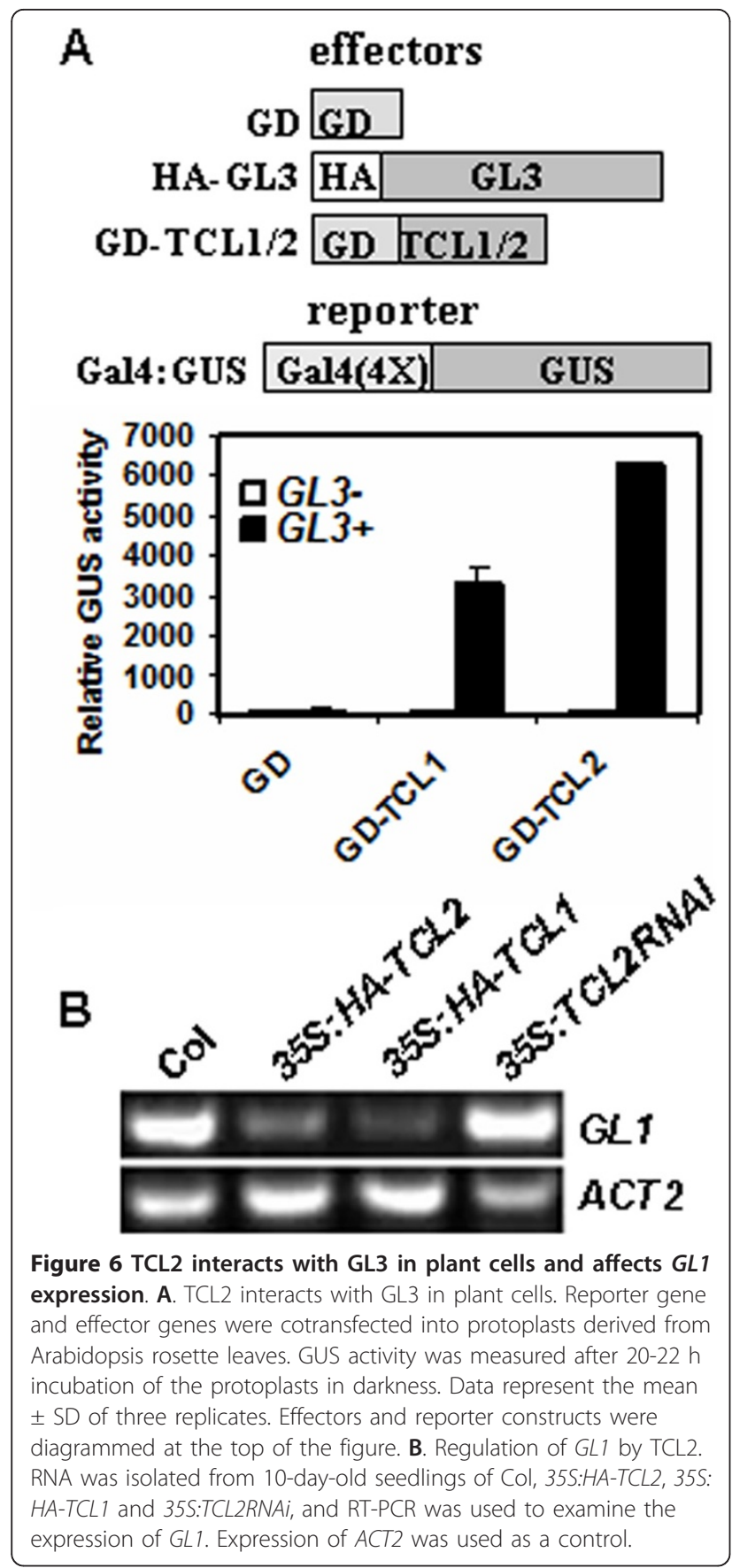

the Gal4 DNA binding elements in the reporter gene [20]. It was also observed that the expression of the reporter gene activated by GD-TCL2 in the presence of GL3 is much higher than that of GD-TCL1 (Figure 6A), implying that the binding affinity between TCL2 and GL3 may be higher than that between TCL1 and GL3.

TCL2 suppresses the expression of GL1

In addition to competing with GL1 for binding GL3, we also reported previously that TCL1 can directly suppress the expression of GL1 [13]. Because TCL2 is more closely related to TCL1 than any other single-repeat MYBs at the amino acid level (Figure 1) and TCL2RNAi lines displayed similar inflorescence and pedicels trichome phenotypes as tcl1 mutants, we then tested if TCL2 also affects the expression of GL1. As shown in Figure 6B, expression level of $G L 1$ is dramatically reduced in $35 S$ : HA-TCL2 seedlings, similar to that in 35S:HA-TCL1 seedlings, suggesting that TCL2 can also suppress the expression of GL1.

\section{Neither GL1-GL3 activator complex nor SPLs alone activates $T C L 2$}

Available evidence suggested that TTG1-GL3-GL1 activator complex activates both GL2 and some single-repeat MYB genes. Our previous reporter showed that GL1 and GL3 are required and sufficient to activate GL2 and a subset of single-repeat MYB genes including TRY, CPC, ETC1 and ETC3, but not ETC2 and TCL1[19,20]. To test if TCL2 is regulated by GL1-GL3 complex, GL1 and GL3 were co-transfected into protoplasts and RT-PCR was used to examine the expression of TCL2. Expression of $C P C$ was used as a positive control. As expected, $C P C$ was strongly induced by GL1 + GL3. However, such activation was not observed for TCL2 (Figure 7A).

We wanted to further investigate the molecular mechanism controlling the activation of TCL2 transcription. Recently, it has been showed that TCL1 is directly activated by MIR156 directed SPLs [21]. In order to explore the possibility that expression of TCL2 may also be controlled by MIR156 directed SPLs, we tested the expression of TCL2 in 35S:MIR156 plant. Indeed, we

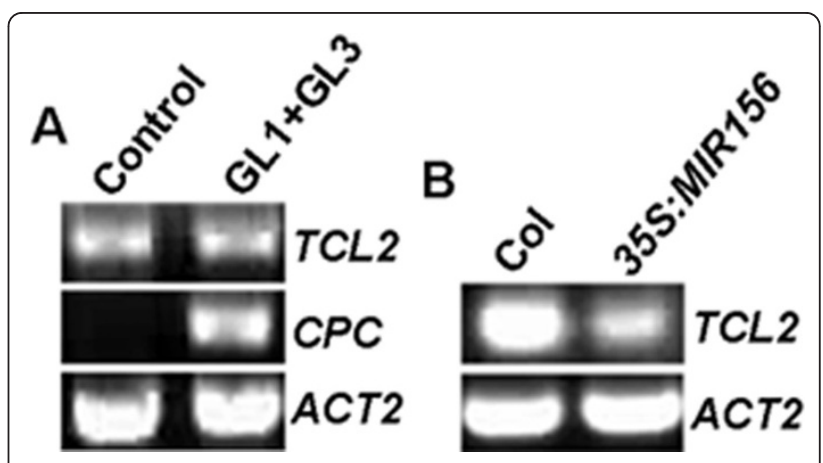

Figure 7 Regulation of TCL2. A. Expression of TCL2 is not regulated by GL1-GL3 activator complex. GL1 and GL3 were cotransfected into protoplasts derived from Arabidopsis rosette leaves, RNA was isolated from transfected protoplasts after 20-22 h incubation in darkness, and RT-PCR was used to examine the expression of TCL2 and CPC. Expression of ACT2 was used as a control. B. Expression of TCL2 is down-regulated in 35S:MIR156 plants. RNA was isolated from 10-day-old seedlings of Col and 35S: MIR156, and RT-PCR was used to examine the expression of TCL2. Expression of ACT2 was used as a control. 
found that the expression level of TCL2 in 35S:MIR156 plant was dramatically reduced (Figure 7B), similar to that of TCL1 [21]. To further test if SPLs can directly active TCL2, we cloned five SPL genes including SPL3, SPL9, SPL10, SPL13 and SPL15, transiently expressed them in protoplasts, and then used RT-PCR to examine the expression of TCL2. The five SPLs were chosen because all of them but SPL15 have been shown to regulate trichome formation [21], while SPL15 is closely related to SPL9 [24]. To our surprise, no significant changes were observed (data not shown). Since overexpression of SPLs in plants has already been shown to be able to induce the expression of TCL1, and SPL9 has been shown to directly bind to the promoter region of TCL1 [21], we test if the expression of TCL1 in protoplasts transfected with SPLs is affected. However, we did not see any significant changes (data not shown). These results suggested that SPLs alone may not be sufficient to activate the expression of TCL1. To explore this possibility further, all five SPLs cloned were fused with GD and co-transfected with Gal4:GUS reporter gene into protoplasts, then GUS activities were assays. Indeed, none of the five SPLs tested could activate the reporter gene (data not shown). These results indicated that although SPLs can bind directly to the promoter region of TCL1, they might require co-activators to regulate the expression of their target genes, including TCL1, TRY and possibly, TCL2.

\section{Discussion}

\section{TCL2 is a negative regulator of trichome formation}

In this study we analyzed the function of TCL2, a previously uncharacterized single-repeat R3 MYB transcription factor in trichome formation in Arabidopsis. We showed that TCL2, like TCL1, TRY, CPC, ETC1, ETC2 and ETC3, act as a negative regulator for trichome formation. Overexpression of TCL2 under the control of the CaMV $35 S$ promoter resulted in a glabrous phenotype (Figure 4). Knockdown of TCL2 via RNAi (TCL2RNAi) resulted in ectopic trichome formation on the inflorescence stems and pedicels (Figure 4), while the number and patterning of trichomes on leaves were largely unaffected, a phenotype similar to that of $t c l 1$ [13]. These results suggest that TCL2 negatively regulates trichome formation and that TCL2 may have overlapping function with TCL1 in controlling trichome formation on the inflorescence stems and pedicels.

We also showed that expression of TCL2 under the control of the TCL1 promoter in a tcl1 mutant background $\left(P_{T C L 1}: H A-T C L 2 / t c l 1\right)$ could only partially rescue the trichome phenotype of the $t c l 1$ mutant. Trichomes were still present on the inflorescence stems and pedicels of $P_{T C L 1}$ : TCL2/tcl1 plants, although the frequency was significantly reduced (Figure 5). On the other hand, expression of
TCL1 under the control of the TCL1 promoter in tcl1 mutant background fully rescued the trichome phenotype of the tcl1 mutant [13]. These results implied that TCL2 and TCL1 may function redundantly to control trichome development on inflorescence stems and pedicels but their function may not fully interchangeable.

Although transcript of TCL2 is detectable in roots (Figure 2), all the mutants tested including TCL2 overexpressor, TCL2RNAi and $P_{T C L 1}$ :TCL2/tcl1 are largely indistinguishable from wild type in term of root hair formation (data not shown). However, we could not rule out the possibility that TCL2 may play a role in regulating root hair formation, a higher order combination of mutations in single repeat MYBs may help clarify the exact role of TCL2 in root formation.

\section{How does TCL2 regulate trichome formation}

Previous research has pointed out that trichome formation is regulated by an activator/inhibitor feedback system $[7,10,19,25]$. TTG1, GL1, and GL3 or EGL3 form an activator complex (TTG1-GL3/EGL3-GL1) that initiates trichome development by activating GL2. The same activator complex also activates some single-repeat R3 MYB transcription factor genes, whose products, in turn, compete with GL1 for binding GL3/EGL3, thus inhibiting the formation of the activator complex. Our previous study suggested that TCL1 could also directly suppress the expression of GL1, providing another negative feedback loop on the regulation of trichome formation [13]. By using a protoplast transfection system, we showed that TCL2 interacts with GL3 (Figure 6A), suggesting that TCL2 could block the formation of the activator complex required for trichome formation (see Additional file 2). RT-PCR results showed that expression of GL1 is dramatically reduced in the plants overexpressing $H A-T C L 2$, indicating the TCL2 can also suppress the expression of GL1. These results suggest that TCL2 negatively regulate trichome formation in a manner similar to that of TCL1 (see Additional file 2). However, we could not rule out the possibility that TCL2 may also regulate trichome formation through other mechanisms, since our recent results showed that single-repeat MYBs may regulate trichome formation independent of GL2 [26].

\section{Regulation of TCL2}

Available evidence supports that TTG1-GL3/EGL3-GL1 activator complex plays an important role in regulating trichome formation. Recently, several phytohormones, such as jasmonic acid (JA), gibberellins (GAs) and cytokinins are found to be involved in the promotion of trichome formation, and this promotion is mediated, at least in part, by transcriptional regulation of the components of TTG1GL3/EGL3-GL1 complex [27-30], providing further support for the critical role of the activator complex in the 
regulation of trichome formation. In addition to regulating the expression of GL2 to promote trichome formation, the activator complex also activates some single-repeat MYB genes to provide a negative feedback loop. Available evidence suggested that the transcription of $C P C, T R Y$, ETC1, and ETC3, but not TCL1 and ETC2, is regulated by the proposed activator complex $[19,31]$, implying that other pathways may also be involved in the regulation of single-repeat MYB genes. Recently, it has been showed that MIR156-regulated SPLs, which promote phase transition, directly activate TCL1 and TRY expression through binding to their promoters and that this activation is independent of GL1[21].

Using protoplast transient expression system, we found that the expression of TCL2 is not regulated by TTG1GL3/EGL3-GL1 complex (Figure 7A). Reduced expression of TCL2 in 35S:MIR156 transgenic plants suggested that MIR156-targeted SPLs may also regulate the expression of TCL2 (Figure 7B). However, when expressed in protoplasts, none of the five SPLs tested could induce the expression of neither TCL2 nor TCL1. Further test suggested that SPLs alone could not activate reporter gene expression when recruited to the promoter region of the reporter gene by a fused DNA binding domain. These results indicated that there might be other co-activator working together with SPLs to regulate the expression of TCL1, and possible TCL2 (see Additional file 2). It is also possible that unidentified targets of MIR156 may involve in the regulation of single-repeat MYB genes.

\section{Conclusions}

In this report, we identified TCL2 as a new member of single-repeat MYB transcription factor family, and provided evidence to show that TCL2 is a negative regulator of trichome development. We showed that TCL2 may have overlapping function with TCL1 to regulate trichome formation, but its function is not fully interchangeable with TCL1. Furthermore, we showed that TCL2 interact with GL3 in plant cells, its expression is not regulated by GL3/GL1 activator complex, and that MIR156 is involved in the regulation of TCL2. Finally, we found that although SPLs can bind directly to the promoter region of TCL1, they likely require co-activators to regulate the expression of their target genes, including TCL1, TRY, and possibly, TCL2.

\section{Methods}

\section{Plant materials and growth conditions}

The tcl1 mutant, 35S:MIR156 and 35S:HA-TCL1 transgenic plant are in the Columbia-0 (Col-0) background $[13,21]$. T-DNA insertion lines related to TCL2 were obtained from SALK Institute.

Seedlings used for RT-PCR analysis were grown on $1 / 2$ Murashige \& Skoog (MS) medium with vitamins (plantmedia) and 1\% (w/v) sucrose. Seedlings used for phenotypic analysis were obtained either by plating seeds on $1 / 2$ MS medium or by directly sowing seeds into soil. Plants were grown at $22 \mathrm{C}$ with $14 / 10$ hour photoperiod at approximately $120 \mu \mathrm{mol} \mathrm{m} \mathrm{s}^{-2}$.

\section{Constructs}

35S:GD-TCL1, 35S:HA-GL3 have been described previously [13]. To generate HA or GD tagged constructs for TCL2, SPL3, 9, 10, 13 and 15, the full-length open-reading frames (ORF) of corresponding genes were amplified by RT-PCR using RNA from 10-day-old light-grown Arabidopsis seedlings, then the PCR products were cloned in frame with an N-terminal HA or GD tag into the pUC19 vector under the control of the double $35 S$ enhancer promoter of CaMV. The $P_{\text {TCL2 }}: H A-T C L 2-G F P$ was cloned by fusing TCL2 in frame with GFP and then subcloned into the pUG19 vector under the control of the TCL2's own promoter (a fragment that covers the region -1502 to +1 of the start codon of TCL2). For plant transformation, corresponding constructs in $p U G 19$ vector were digested with $E c o R I$, then subcloned into the binary vector $p P Z P 211$ or pPZP221 [32].

To generate TCL2RNAi construct. The TCL2 fragment was amplified by RT-PCR using forward primer 5' -CAACTCGAGGATAACACCAACCGTCT-3' and reverse primer 5'-CAAGAATTCAGGAGGAGAAATAGAGA-3', which contain an XhoI and an EcoRI enzyme cutting site at its 5 '-end respectively. The PCR product was digested by XhoI and EcoRI and cloned into vector pHANNIBAL. The vector contained the CaMV $35 S$ promoter and the nos terminator in sense orientation. The resulting plasmid was named as $p H A N N I B A L-$ TCL2S. The anti-sense fragment was amplified by RTPCR using forward primer 5'-CAATCTAGAGATAACACCAACCGTCT-3' and reverse primer 5'-CAAAA GCTTAGGAGGAGAAATAGAGA-3', which contain an $X b a I$ site and a HindIII enzyme cutting site at its 5 '-end respectively. The PCR product was digested by $X b a I$ and HindIII and cloned into PHANNIBAL-TCL2S plasmid, resulting $p H A N N I B A L-T C L 2 R N A i$ construct. The TCL2RNAi cassette was then removed from pHANNIBAL-TCL2RNA $i$ by digestion with NotI, and subcloned into binary vector $p A R T 27$ for plant transformation.

\section{Phylogenetic analysis}

Full length protein sequences of TRY, CPC, TCL1, TCL2, ETC1, ETC2 and ETC3/CPL3 were used for phylogenetic analysis. Multiple sequence alignment and dendrogram with branch length were generated using CLUSTAL W Multiple Sequence Alignment Program with default settings (http://www.genome.jp/tools-bin/ clustalw). 
Plant transformation and selection of transgenetic plants About five-week-old plants with several mature flowers on the main inflorescence were used to transform with various constructs via Agrobacterium tumefaciens GV3101 by using the floral dip method [33]. Phenotypes of transgenic plants were examined in the T1 generation, and confirmed in T2 up to T4 generations. Up- or down-expression of corresponding genes in related lines was confirmed by RT-PCR. For each construct, at least three independent transgenic lines with similar phenotypes were obtained, and results from a representative line were presented.

\section{Plasmid DNA isolation, protoplast transfection and GUS activity assay}

Plasmid DNA was prepared using the Qiagen EndoFree Plasmid Maxi Kit (http://www.qiagen.com). Protoplast isolation, transfection and the GUS activity assay were performed as described previously [34-36]. Briefly, protoplasts were isolated from rosette leaves of $\sim 4$-week-old plants. Effector and reporter plasmids were co-transfected into protoplasts and incubated under darkness for 20-22 h. GUS activities were measured using a BioTEK Synergy ${ }^{\mathrm{TM}}$ HT microplate reader (http://www.biotek.com). Expression of 35S:luciferase (Luc) was used to normalize the expression of the GUS reporter. Luciferase activities were measured using a microplate luminometer (http://www. turnerdesigns.com) together with the Promega Steady-Glo luciferase assay system (http://www.promega.com/).

\section{Microscopy}

Trichomes were analyzed and photographed as described previously $[13,19]$.

Four-day-old seedlings expressing TCL2-GFP under the control of the TCL2 promoter were used to examine the expression and localization of TCL2-GFP, by using a Leica DM-6000B upright fluorescent microscope with phase and differential interference contrast (DIC), photos were taken using a Leica FW4000 digital camera connected to the microscope (http://www.leica-microsystems.com).

\section{RNA isolation and RT-PCR}

Total RNA was isolated from different parts of plant, seedlings or transfected protoplasts using the RNeasy Plant Mini Kit (QIAGEN, Mississauga, Ontario, Canada). cDNA was synthesized using $2 \mu \mathrm{g}$ total RNA by Oligo(dT)primed reverse transcription using OMNISCRIPT RT Kit (QIAGEN). Some of the primers used for cloning or examining the expression of corresponding genes have been described previously [13,19], and others are as follows. TCL2-specific primers (5'-CATATGGATAACACCAACCGTCT-3' and 5'-GAGCTCTTAAGGAGGAGAA ATAG-3') were used to amplify the full-length ORF of
TCL2. HA-specific (5'-TACCCTTACGATGTTCCT GATTAC-3') and TCL2-specific (5'-GAGCTCTTAAGGAGGAGAAATAG-3') primers were used to check the expression of HA-TCL2. TCL2RNAi-specific primers (5'GTCCTCTTTCACTTTCAAATACCAATG-3' and 5'-CT TAAAGCTTTTATCTTGTCC-3') were used to check the expression of TCL2 in TCL2RNAi transgenic plants.

\section{Additional material}

Additional file 1: Adobe Photoshop, Expression of single repeat
MYB genes in 35S:TCL2RNAi transgenic plants. RNA was isolated from
10-day-old seedlings of wild type and two independent 35S:TCL2RNAi
transgenic lines, and RT-PCR was used to check the expression of single
repeat MYB genes. Note that transcript of ETC1 was not included in the
figure because we were unable to detect the expression of ETC1 in
seedlings. The expression of ACTIN2 (ACT2) was used as a control.
Additional file 2: Adobe Photoshop, Model of the transcription factor
network that controls trichome formation in Arabidopsis. TTG1, GL3/
EGL3 and GL1 form an activator complex, to regulate the transcription of
GL2, and some of the single repeat MYB genes. Single MYB transcription
factors, in turn, compete with GL1 for binding of GL3/EGL3, thus limiting the
formation of the TTG1-GL3-GL1 activator complex. As shown in this study,
TCL2 can intact with GL3, it can also suppress the expression of GL1, thereby
limiting the transcriptional activity of the TTG1-GL3-GL1 activator complex.
Like that of TCL1, transcription of TCL2 is regulated by SPLS, however,
unknown co-activator may required for the transcriptional activation.
Question marks indicate unknown regulators or unclear processes.

\section{Acknowledgements}

We thank Dr. Jia-Wei Wang and Dr. Detlef Weigel for sharing seeds of the 35S:MIR156 transgenic plants, ABRC for T-DNA insertion lines, and Dr. Shawn Mansfield for providing PHANNIBAL and PART27 vectors. Support for this research was provided by the National Natural Science Foundation of China (grant no. 31170262) and the startup funds from Northeast Normal University to S. W., and by the Laboratory Directed Research and Development Program of Oak Ridge National Laboratory. Oak Ridge National Laboratory is managed by UT-Battelle, LLC, for the U.S. Department of Energy under contract DE-AC05-000R22725.

\section{Author details}

${ }^{1}$ College of Life Sciences, Nanjing Agricultural University, Nanjing 210095 , China. ${ }^{2}$ Key Laboratory of Molecular Epigenetics of MOE and Institute of Genetics \& Cytology, Northeast Normal University, Changchun 130024, China. ${ }^{3}$ Biosciences Division, Oak Ridge National Laboratory, Oak Ridge, TN 37831, USA. ${ }^{4}$ Department of Botany, University of British Columbia, Vancouver, BC V6T 1Z4, Canada.

\section{Authors' contributions}

LG carried out most of the experiments. SW designed the experiments and carried out some of the experiments. LG and SW drafted the manuscript. KX and J-GC conceived of the study, participated in its design and helped to edit the manuscript. All authors read and approved the final manuscript.

\section{Competing interests}

The authors declare that they have no competing interests.

Received: 8 May 2011 Accepted: 15 December 2011

Published: 15 December 2011

\section{References}

1. Oppenheimer DG, Herman PL, Sivakumaran S, Esch J, Marks MD: A myb gene required for leaf trichome differentiation in Arabidopsis is expressed in stipules. Cell 1991, 67:483-493. 
2. Payne $C T$, Zhang F, Lloyd AM: GL3 encodes a bHLH protein that regulates trichome development in Arabidopsis through interaction with GL1 and TTG1. Genetics 2000, 156:1349-1362.

3. Zhang F, Gonzalez A, Zhao M, Payne CT, Lloyd AM: A network of redundant bHLH proteins functions in all TTG1-dependent pathways of Arabidopsis. Development 2003, 30:4859-4869.

4. Galway ME, Masucci JD, Lloyd AM, Walbot V, Davis RW, Schiefelbein JW: The $\Pi G$ gene is required to specify epidermal cell fate and cell patterning in the Arabidopsis root. Dev Biol 1994, 166:740-754.

5. Walker $A R$, Davison PA, Bolognesi-Winfield AC, James $C M$, Srinivasan $N$, Blundell TL, Esch JJ, Marks MD, Gray JC: The TRANSPARENT TESTA GLABRA1 locus, which regulates trichome differentiation and anthocyanin biosynthesis in Arabidopsis, encodes a WD40 repeat protein. Plant Cell 1999, 11:1337-1350.

6. Rerie WG, Feldmann KA, Marks MD: The GLABRA2 gene encodes a homeo domain protein required for normal trichome development in Arabidopsis. Genes Dev 1994, 8:1388-1399.

7. Pesch M, Hülskamp M: Creating a two-dimensional pattern de novo during Arabidopsis trichome and root hair initiation. Curr Opin Genet Dev 2004, 14:422-427.

8. Pesch M, Hülskamp M: One, two, three...models for trichome patterning in Arabidopsis? Curr Opin Plant Biol 2009, 12:587-592.

9. Schnittger A, Folkers U, Schwab B, Jürgens G, Hülskamp M: Generation of a spacing pattern: The role of TRIPTYCHON in trichome patterning in Arabidopsis. Plant Cell 1999, 11:1105-1116.

10. Schellmann S, Schnittger A, Kirik V, Wada T, Okada K, Beermann A, Thumfahrt J, Jürgens G, Hülskamp M: TRIPTYCHON and CAPRICE mediate lateral inhibition during trichome and root hair patterning in Arabidopsis. EMBO J 2002, 21:5036-5046.

11. Wada T, Tachibana T, Shimura Y, Okada K: Epidermal cell differentiation in Arabidopsis determined by a Myb homolog, CPC. Science 1997, 277:1113-1116

12. Wada T, Kurata T, Tominaga R, Koshino-Kimura Y, Tachibana T, Goto K, Marks MD, Shimura Y, Okada K: Role of a positive regulator of root hair development, CAPRICE, in Arabidopsis root epidermal cell differentiation. Development 2002, 129:5409-5419.

13. Wang S, Kwak SH, Zeng Q, Ellis BE, Chen XY, Schiefelbein J, Chen JG: TRICHOMELESS1 regulates trichome patterning by suppressing GLABRA1 in Arabidopsis. Development 2007, 134:3873-3882.

14. Esch JJ, Chen MA, Hillestad M, Marks MD: Comparison of TRY and the closely related At1g01380 gene in controlling Arabidopsis trichome patterning. Plant J 2004, 40:860-869.

15. Kirik V, Simon M, Hülskamp M, Schiefelbein J: The ENHANCER OF TRY AND CPC1 gene acts redundantly with TRIPTYCHON and CAPRICE in trichome and root hair cell patterning in Arabidopsis. Dev Biol 2004, 268:506-513.

16. Kirik V, Simon M, Wester K, Schiefelbein J, Hülskamp M: ENHANCER of TRY and CPC 2(ETC2) reveals redundancy in the region-specific control of trichome development of Arabidopsis. Plant Mol Biol 2004, 55:389-398.

17. Tominaga R, Iwata M, Sano R, Inoue K, Okada K, Wada T: Arabidopsis CAPRICE-LIKE MYB 3 (CPL3) controls endoreduplication and flowering development in addition to trichome and root hair formation. Development 2008, 135:1335-1345.

18. Wester K, Digiuni S, Geier F, Timmer J, Fleck C, Hülskamp M: Functional diversity of R3 single-repeat genes in trichome development. Development 2009, 136:1487-1496

19. Wang S, Hubbard L, Chang Y, Guo J, Schiefelbein J, Chen JG: Comprehensive analysis of single-repeat R3 MYB proteins in epidermal cell patterning and their transcriptional regulation in Arabidopsis. BMC Plant Biol 2008, 8:81.

20. Wang S, Chen JG: Arabidopsis transient expression analysis reveals that activation of GLABRA2 may require concurrent binding of GLABRA1 and GLABRA3 to the promoter of GLABRA2. Plant Cell Physiol 2008, 49:1792-804

21. Yu N, Cai WJ, Wang S, Shan CM, Wang LJ, Chen XY: Temporal Control of Trichome Distribution by MicroRNA156-Targeted SPL Genes in Arabidopsis thaliana. Plant Cell 2010, 22:2322-2335

22. Zimmermann IM, Heim MA, Weisshaar B, Uhrig JF: Comprehensive identification of Arabidopsis thaliana MYB transcription factors interacting with R/B-like BHLH proteins. Plant J 2004, 40:22-34

23. Kurata T, Ishida T, Kawabata-Awai C, Noguchi M, Hattori S, Sano R, Nagasaka R, Tominaga R, Koshino-Kimura Y, Kato T: Cell-to-cell movement of the CAPRICE protein in Arabidopsis root epidermal cell differentiation. Development 2005, 132:5387-5398.

24. Schwarz S, Grande AV, Bujdoso N, Saedler H, Huijser P: The microRNA regulated SBP-box genes SPL9 and SPL15 control shoot maturation in Arabidopsis. Plant Mol Biol 2008, 67:183-195.

25. Larkin JC, Brown ML, Schiefelbein J: How do cells know what they want to be when they grow up? Lessons from epidermal patterning in Arabidopsis. Annu Rev Plant Biol 2003, 54:403-430.

26. Wang S, Barron C, Schiefelbein J, Chen JG: Distinct relationships between GLABRA2 and single-repeat R3 MYB transcription factors in the regulation of trichome and root hair patterning in Arabidopsis. New Phytolo 2010, 185:387-400.

27. Gan Y, Liu C, Yu H, Broun P: Integration of cytokinin and gibberellin signalling by Arabidopsis transcription factors GIS, ZFP8 and GIS2 in the regulation of epidermal cell fate. Development 2007, 134:2073-2081.

28. Maes L, Inzé D, Goossens A: Functional specialization of the TRANSPARENT TESTA GLABRA1 network allows differential hormonal control of laminal and marginal trichome initiation in Arabidopsis rosette leaves. Plant Physiol 2008, 148:1453-64.

29. Yoshida Y, Sano R, Wada T, Takabayashi J, Okada K: Jasmonic acid control of GLABRA3 links inducible defense and trichome patterning in Arabidopsis. Development 2009, 136:1039-1048.

30. Maes $L$, Goossens A: Hormone-mediated promotion of trichome initiation in plants is conserved but utilizes species- and trichome-specific regulatory mechanisms. Plant Sign Beha 2010, 5:205-207.

31. Morohashi K, Zhao M, Yang M, Read B, Lloyd A, Lamb R, Grotewold E: Participation of the Arabidopsis bHLH factor GL3 in trichome initiation regulatory events. Plant Physiol 2007, 145:736-746.

32. Hajdukiewicz P, Svab Z, Maliga P: The small, versatile pPZP family of Agrobacterium binary vectors for plant transformation. Plant Mol Biol 1994, 25:989-994.

33. Clough SJ, Bent AF: Floral dip: a simplified method for Agrobacteriummediated transformation of Arabidopsis thaliana. Plant J 1998, 16:736-743.

34. Tiwari SB, Hagen G, Guilfoyle TJ: The roles of auxin response factor domains in auxin-responsive transcription. Plant Cell 2003, 15:533-543.

35. Wang S, Chang Y, Guo J, Chen JG: Arabidopsis Ovate Family Protein 1 is a transcriptional repressor that suppresses cell elongation. Plant J 2007, 50:858-872.

36. Wang S, Tiwari SB, Hagen G, Guilfoyle TJ: AUXIN RESPONSE FACTOR7 restores the expression of auxin-responsive genes in mutant Arabidopsis leaf mesophyll protoplasts. Plant Cell 2005, 17:1979-1993.

doi:10.1186/1471-2229-11-176

Cite this article as: Gan et al:: Functional characterization of

TRICHOMELESS2, a new single-repeat R3 MYB transcription factor in the regulation of trichome patterning in Arabidopsis. BMC Plant Biology 2011 11:176

\section{Submit your next manuscript to BioMed Central and take full advantage of:}

- Convenient online submission

- Thorough peer review

- No space constraints or color figure charges

- Immediate publication on acceptance

- Inclusion in PubMed, CAS, Scopus and Google Scholar

- Research which is freely available for redistribution 